\title{
Symmetric Pulsed Anodizing of Aluminum Foil for Aluminum \\ Electrolytic Capacitors
}

Xiang Li, Baige Lin, Lilong Xiong, Xianfeng Du*

Xi'an Key Laboratory of Sustainable Energy Materials Chemistry, MOE Key

Laboratory for None-quilibrium Synthesis and Modulation of Condensed Matter,

School of Science, Xi'an Jiaotong University, Xi'an 710049, P. R. China.

E-mail : xianfengdu@mail.xjtu.edu.cn

\section{- SUPPORTING INFORMATION}

Figure S1. Schematic diagram of (a) etched foil and (b) DC and (c) SPC anodizing devices.

Figure S2. The output current and voltage waveform of pulse anodizing.

Figure S3. The electrochemical performance of anode foil under different (a) formation voltages (b) current density and (c) pulse frequency.

Figure S4. The schematic diagram of energy consumption.

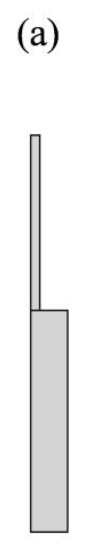

(b)

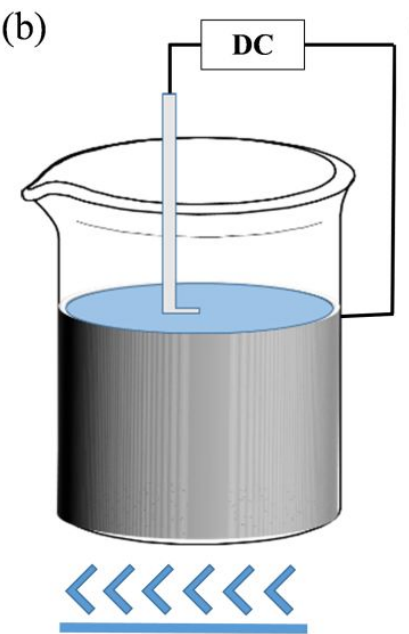

Heating System (c)

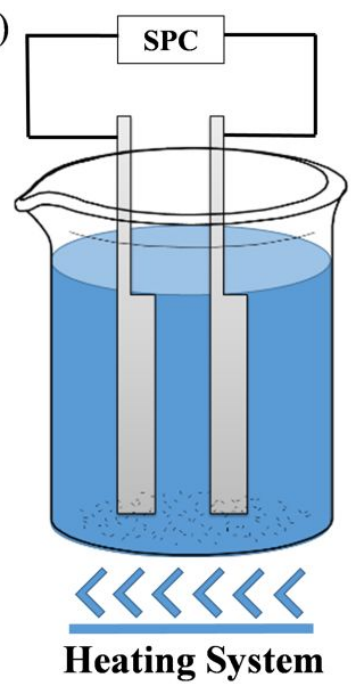

Figure S1. Schematic diagram of (a) etched foil (b) DC and (c) SPC anodizing devices. 


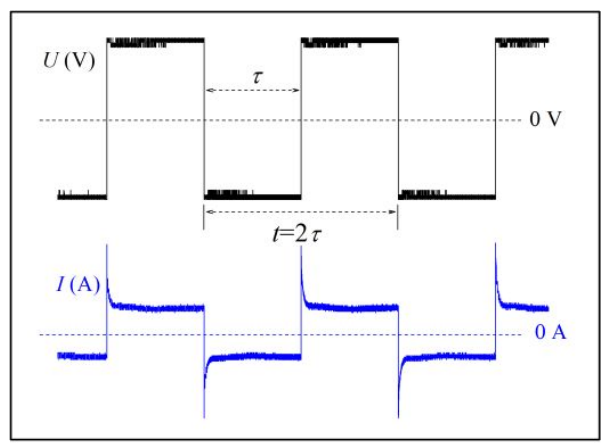

Time(s)

Figure S2 The output current and voltage waveform of pulse anodizing. 

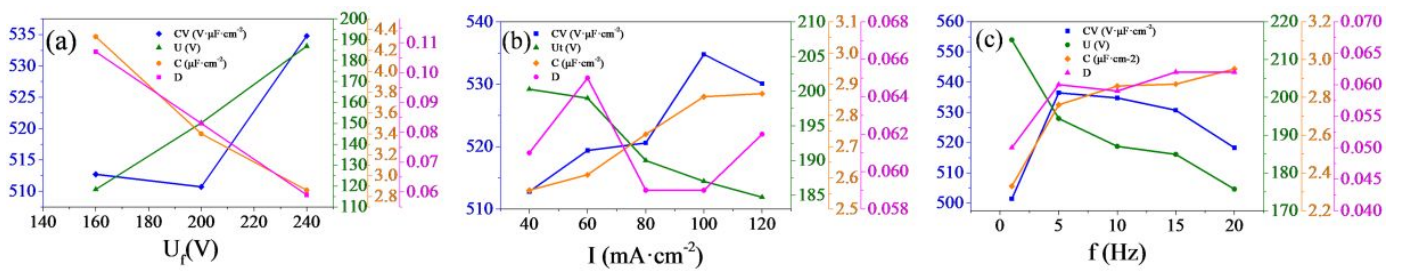

Figure S3 The electrochemical performance of anode foil under different (a) formation voltages (b) current density and (c) pulse frequency. 


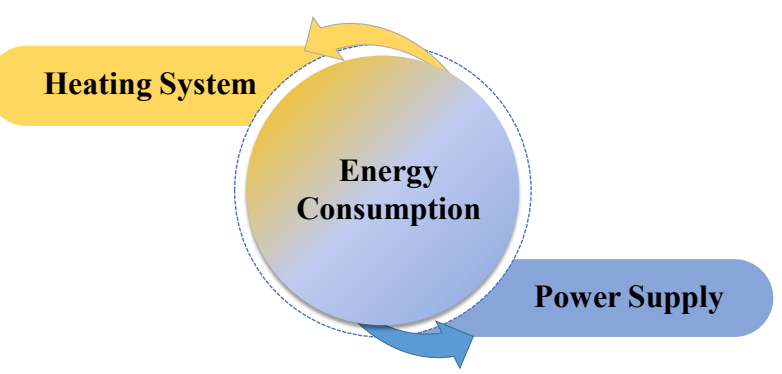

Figure S4 The schematic diagram of energy consumption. 